\title{
SORÇÃO DO HERBICIDA 2,4-D EM SOLOS BRASILEIROS
}

\author{
CLAUDIO A. SPADOTTO * \\ MARCUS B. MATALLO ** \\ MARCO ANTONIO F. GOMES ***
}

\begin{abstract}
Neste trabalho procurou-se gerar e discutir dados sobre sorção de pesticidas ácidos em solos brasileiros, considerando sua dependência do $\mathrm{pH}$. Amostras de solos foram coletadas em diferentes regiões do Brasil e suas propriedades determinadas (\% de argila, \% de $\mathrm{CO}$ e pH). Valores do coeficiente de sorção do herbicida 2,4-D foram determinados em laboratório usando teste em equilíbrio padrão e cromatografia a líquido de alta eficiência (CLAE). $\mathrm{O}$ coeficiente de sorção $(\mathrm{Kd})$ foi estimado e o coeficiente de sorção normalizado pela fração de carbono orgânico do solo (Koc) calculado para cada solo. Os resultados de Koc variaram entre 21,3 e $306,5 \mathrm{~mL} / \mathrm{g}$, sendo que dados de Koc encontrados na literatura internacional estão entre 20 e $60 \mathrm{~mL} / \mathrm{g}$. Observou-se tendência de aumento dos valores de $\mathrm{Kd}$ em solos com maiores teores de carbono orgânico. No entanto, houve grande variação nos valores de Koc entre solos da mesma classe. Adicionalmente, os efeitos do $\mathrm{pH}$ na sorção do 2,4-D foram estudados em Latossolo. Os valores de $\mathrm{Kd}$ e Koc diminuíram com o aumento do $\mathrm{pH}$. A sorção do 2,4-D, como composto ácido, é principalmente determinada pela sua dissociação. Entretanto, a dissociação do 2,4-D pode não ser suficiente para explicar a variação em Kd (ou Koc) em função do pH. Parece que a sorção das formas aniônicas de pesticidas ácidos tem sido superestimada e o efeito da dissociação da matéria orgânica, com mudanças no pH do solo, tem sido subestimada.
\end{abstract}

PALAVRAS-CHAVE: PESTICIDA-SORÇÃO; SOLO; MATÉRIA ORGÂNICA; 2,4-D.

* Engenheiro Agrônomo, PhD em Ciência de Solo-Água, Pesquisador da Embrapa Meio Ambiente, Jaguariúna, SP (e-mail: spadotto@cnpma.embrapa.br).

** Engenheiro Agrônomo, DSc em Agronomia, Pesquisador do Instituto Biológico, Campinas, SP.

*** Geólogo, DSc em Solos, Pesquisador da Embrapa Meio Ambiente, Jaguariúna, SP. 


\section{INTRODUÇÃO}

Usa-se o termo sorção para diversas associações possíveis entre pesticidas e colóides do solo (adsorção, absorção, etc). Mecanismos múltiplos de sorção podem estar envolvidos na associação de determinado pesticida com sistema heterogêneo como o solo. Pesquisas extensivas têm sido conduzidas tratando da sorção de pesticidas e várias revisões sobre o tópico têm sido publicadas. Informações do Pesticide Manual (TOMLIN, 2000) revelam que a sorção dependente do $\mathrm{pH}$ pode ser relevante para grande parte dos pesticidas registrados no Brasil. Além disso, essa dependência pode também ser valiosa para produtos de degradação de outros pesticidas.

A sorção de pesticidas -ácidos aos colóides orgânicos do solo depende do pH, sendo maior sob condições ácidas quando os pesticidas são sorvidos na forma molecular (neutra). Tal dependência tem sido reportada para muitos pesticidas ácidos, incluindo Bentazon (ABERNATHY e WAX, 1973; GREY et al., 1996; LI et al., 2003), Clorimuron (GOETZ et al., 1989), Clorosulfuron (SHEA, 1986; WALKER et al., 1989), Metasulfuron-metil (WALKER et al., 1989) e Picloram (NEARPASS, 1976). Sabe-se que a sorção exerce grande impacto na distribuição, biodisponibilidade e persistência de pesticidas no ambiente.

O 2,4-D [2,4-diclorofenoxiacético] apresenta propriedades interessantes para o estudo de sua sorção em solos brasileiros. Herbicida hormonal do grupo dos fenoxiacéticos, desenvolvido há mais de 60 anos, o 2,4-D ainda é amplamente usado no Brasil e no mundo para controle de plantas daninhas de folhas largas.

Estudos realizados anteriormente mostraram que o coeficiente de sorção normalizado para carbono orgânico do solo (Koc) do 2,4-D está entre 20 e $60 \mathrm{~mL} / \mathrm{g}$. Além disso, mais de $90 \%$ da sua sorção ocorre dentro de $2 \mathrm{~h}$ em condições de laboratório. HORNSBY et al. (1996), considerando várias referências, selecionou 10 dias como valor de meia-vida do 2,4-D. OGRAM et al. (1985) sugeriram que a degradação microbiana de 2,4-D ocorre apenas na fase em solução, e não no herbicida sorvido.

A dependência da sorção ao $\mathrm{pH}$ do solo tem sido reportada há anos para muitos pesticidas ácidos, como o herbicida 2,4-D. Entretanto, o tema vem ganhando destaque devido aos questionamentos sobre o comportamento ambiental dos pesticidas, particularmente sobre sorção em solos com cargas variáveis, como muitos solos agrícolas do Brasil.

O presente trabalho foi realizado com o objetivo de suprir, em parte, a carência de dados sobre sorção de pesticidas/herbicidas em diferentes solos brasileiros, sendo incluída discussão sobre possíveis mecanismos de sorção.

\section{MATERIAL E MÉTODOS}

Amostras de solos foram coletadas em diferentes regiões do Brasil e suas propriedades determinadas (Tabela 1) de acordo com métodos da EMBRAPA (1997) e de CAMARGO et al. (1986).

O herbicida 2,4-D foi escolhido como pesticida ácido padrão, o qual pode apresentar-se nas formas molecular e aniônica nos solos. Devido ao seu baixo valor de constante de dissociação ( $\mathrm{pKa}=$ 2,8), o 2,4-D encontra-se predominantemente na forma aniônica na faixa comum de $\mathrm{pH}$ dos solos.

Valores de coeficiente de sorção de 2,4-D [2,4-diclorofenoxiacético; CAS RN: 94-75-7] foram determinados em laboratório, usando teste em equilíbrio padrão, com $10 \mathrm{mg}$ de 2,4-D analítico por litro de solução de $\mathrm{CaCl}_{2}$ 0,01 M. Uma alíquota de $10 \mathrm{~mL}$ da solução foi adicionada a $5 \mathrm{~g}$ de solo em tubo de centrífuga, agitando-se por $24 \mathrm{~h}$ a cerca de $25^{\circ} \mathrm{C}$. Após o período de equilíbrio, as suspensões foram cetrifugadas por $20 \mathrm{~min}$ a $10000 \mathrm{rpm}$ e o sobrenadante filtrado em filtro de $0,45 \mu \mathrm{m}$. A quantidade do herbicida no sobrenadante foi determinada por cromatografia a líquido de alta eficiência (coluna $C_{18}$ de 3,9 por $150 \mathrm{~mm}$; diâmetro de partícula de $5 \mu \mathrm{m}$, Waters, Milford, MA), com injeções do sobrenadante e da solução padrão feitas em duplicata. A fase móvel foi metanol:água $(70: 30 \mathrm{v} / \mathrm{v})$ e cloreto de tetrabutilamônio a 0,01 M, com detecção a $228 \mathrm{~nm}$. 


\section{TABELA 1 - PROPRIEDADES DOS SOLOS ESTUDADOS}

\begin{tabular}{|c|c|c|c|}
\hline Solo & Argila (\%) & $\mathrm{CO}(\%)$ & $\mathrm{pH}\left(\mathrm{CaCl}_{2}\right)$ \\
\hline Latossolo 1 & 50 & 1,1 & 5,0 \\
\hline Latossolo 2 & 41 & 1,5 & 5,7 \\
\hline Latossolo 3 & 13 & 0,8 & 6,3 \\
\hline Latossolo 4 & 61 & 1,4 & 5,7 \\
\hline Latossolo 5 & 54 & 1,5 & 5,8 \\
\hline Latossolo 6 & 51 & 1,5 & 5,8 \\
\hline Latossolo 7 & 24 & 1,3 & 6,5 \\
\hline Latossolo 8 & 28 & 1,2 & 5,6 \\
\hline Latossolo 9 & 64 & 1,6 & 4,9 \\
\hline Alissolo 1 & 19 & 1,2 & 4,6 \\
\hline Alissolo 2 & 10 & 0,5 & 5,0 \\
\hline Alissolo 3 & 41 & 1,3 & 5,5 \\
\hline Alissolo 4 & 27 & 1,1 & 6,6 \\
\hline Alissolo 5 & 16 & 1,1 & 5,6 \\
\hline Nitossolo 1 & 52 & 1,6 & 5,3 \\
\hline Nitossolo 2 & 42 & 1,3 & 6,1 \\
\hline Espodossolo 1 & 2 & 1,5 & 4,8 \\
\hline Espodossolo 2 & 5 & 0,1 & 6,4 \\
\hline Gleissolo & 39 & 1,4 & 6,0 \\
\hline
\end{tabular}

CO = Teor de carbono orgânico no solo.

A sorção em equilíbrio foi calculada como a diferença de concentração entre a solução inicial e a solução em equilíbrio com o solo, em uma única concentração da solução para cada solo. Assumindo a sorção como processo instantâneo, reversível e linear, estimou-se o valor de coeficiente de sorção (Kd) aparente pela equação 1:

$$
\mathrm{Kd}=\mathrm{S} / \mathrm{C}
$$

na qual: $S=$ massa do herbicida sorvido por massa de solo $(\mu \mathrm{g} / \mathrm{g})$ e $C$ = concentração do herbicida na solução em equilíbrio $(\mu \mathrm{g} / \mathrm{mL})$.

O valor de coeficiente de sorção normalizado pela fração de carbono orgânico do solo foi calculado pela equação 2:

$$
\mathrm{KoC}=\mathrm{Kd} / \mathrm{OC}
$$


na qual: OC representa a fração de carbono orgânico de cada solo.

Adicionalmente, o Latossolo coletado na região de Ribeirão Preto, São Paulo (Latossolo 9 na Tabela 1) foi selecionado para o estudo dos efeitos do pH na sorção do 2,4-D, devido ao seu alto teor de argila (64\%) e predominância de minerais com carga variável.

As mudanças no $\mathrm{pH}$ foram promovidas pela adição de ácido clorídrico $[\mathrm{HCl}] 1 \mathrm{M}$, ou hidróxido de cálcio $\left[\mathrm{Ca}(\mathrm{OH})_{2}\right] 1 \mathrm{M}$, visando produzir valores tanto abaixo como acima do $\mathrm{pH}$ natural do solo. As subamostras de solo foram umedecidas até a capacidade de campo, aguardando-se 6 semanas para equilíbrio do $\mathrm{pH}$. Cada amostra de solo foi umedecida novamente até a capacidade de campo a cada 2 dias. Os coeficientes de sorção foram determinados mediante teste em equilíbrio padrão, conforme descrito anteriormente.

\section{RESULTADOS E DISCUSSÃO}

Os valores de Koc para 9 Latossolos, 5 Alissolos, 2 Nitossolos, 2 Espodossolos e 1 Gleissolo estão apresentados na Tabela 2. Os resultados variaram entre 21,3 e $306,5 \mathrm{~mL} / \mathrm{g}$, sendo que dados de Koc encontrados na literatura internacional enquadram-se na faixa entre 20 e $60 \mathrm{~mL} / \mathrm{g}$ (HORNSBY et al., 1996).

Observou-se tendência ao aumento nos valores do coeficiente de sorção (Kd) em solos com maiores teores de carbono orgânico (CO). No entanto, verificou-se grande variação nos valores do coeficiente de sorção já normalizado para o carbono orgânico do solo (Koc), mesmo entre os solos da mesma classe.

Várias propriedades dos solos têm sido identificadas pelos efeitos que provocam no mecanismo e no grau de sorção de pesticidas. Entretanto, a predominância da sorção de compostos orgânicos não-iônicos na matéria orgânica do solo tem sido extensivamente documentada. Tal fato tem fundamentado o uso do coeficiente de sorção normalizado pela fração de carbono orgânico do solo (Koc). No entanto, conforme observado por STEVENSON (1982), SENESI (1992) e ALMENDROS (1995), os mecanismos envolvidos na sorção de solutos orgânicos na matéria orgânica não têm sido claramente demonstrados. Propriedades da matéria orgânica do solo afetam a sorção de moléculas orgânicas (WERSHAW, 1986). A matéria orgânica é, na verdade, uma mistura complexa e heterogênea de substâncias (STEVENSON, 1982; HAYES, 1985), que pode também estar associada com aluminosilicatos, hidróxidos de alumínio e de ferro e outros constituintes inorgânicos no solo.

Várias hipóteses sobre a estrutura da matéria orgânica têm sido propostas (SCHNITZER e KHAN, 1978; STEVENSON, 1982). Durante a formação da matéria orgânica, diferenças na estrutura e nas características podem surgir em função de condições locais. Rearranjamentos e reações dentro da estrutura da matéria orgânica também continuam ocorrendo depois da sua formação no solo (STEVENSON, 1982; HAIDER, 1992). Assim, mesmo o coeficiente de sorção normalizado para carbono orgânico do solo (Koc) medido para determinado pesticida pode variar consideravelmente entre os solos (SCHEUNERT, 1992). Além disso, tem sido sugerido que estudos de sorção deveriam também considerar, entre outros fatores, a influência das condições físico-químicas do solo sobre a polaridade e a acessibilidade de diferentes grupos funcionais da matéria orgânica (STEVENSON, 1982; SCHNITZER, 1991; SENESI, 1992). O pH é o fator que pode exercer influência particularmente marcante na sorção de pesticidas ácidos, porque a extensão da sorção pode diferir grandemente entre as formas aniônicas e neutras de tais compostos (GRAHAM-BRYCE, 1981).

A Tabela 3 apresenta os valores de Koc do 2,4-D em Latossolo da região de Ribeirão Preto (SP), cujo $\mathrm{pH}$ foi alterado em laboratório a partir do seu valor original. 
TABELA 2 - VALORES DO COEFICIENTE DE SORÇÃO DO 2,4-D NOS SOLOS ESTUDADOS

\begin{tabular}{|c|c|c|}
\hline Solo & $\mathrm{Kd}(\mathrm{mL} / \mathrm{g})$ & $\mathrm{Koc}(\mathrm{mL} / \mathrm{g})$ \\
\hline Latossolo 1 & 0,94 & 82,0 \\
\hline Latossolo 2 & 0,81 & 53,7 \\
\hline Latossolo 3 & 0,36 & 42,9 \\
\hline Latossolo 4 & 1,17 & 80,6 \\
\hline Latossolo 5 & 0,49 & 32,3 \\
\hline Latossolo 6 & 0,91 & 60,3 \\
\hline Latossolo 7 & 0,90 & 70,7 \\
\hline Latossolo 8 & 0,31 & 26,7 \\
\hline Latossolo 9 & 2,10 & 131,3 \\
\hline Alissolo 1 & 1,07 & 89,1 \\
\hline Alissolo 2 & 0,13 & 27,8 \\
\hline Alissolo 3 & 0,40 & 31,1 \\
\hline Alissolo 4 & 0,43 & 40,9 \\
\hline Alissolo 5 & 0,23 & 21,3 \\
\hline Nitossolo 1 & 1,60 & 102,3 \\
\hline Nitossolo 2 & 1,29 & 99,4 \\
\hline Espodossolo 1 & 4,63 & 306,5 \\
\hline Espodossolo 2 & 0,97 & 98,8 \\
\hline Gleissolo & 1,02 & 70,0 \\
\hline
\end{tabular}

$\mathrm{Kd}=$ Coeficiente de sorção.

Koc = Coeficiente de sorção normalizado para carbono orgânico do solo.

\section{TABELA 3 - VALORES DOS COEFICIENTES DE SORÇÃO DO 2,4-D EM LATOSSOLO COM PH} ALTERADO

\begin{tabular}{ccc}
\hline $\mathbf{p H}$ & $\mathrm{Kd}(\mathbf{m L} / \mathbf{g})$ & $\mathrm{Koc}(\mathbf{m} \mathbf{L} / \mathbf{g})$ \\
\hline 4,0 & 7,8 & 487,5 \\
4,7 & 2,7 & 168,8 \\
4,9 & 2,1 & 131,3 \\
5,6 & 1,1 & 68,8 \\
6,2 & 0,4 & 25,0 \\
\hline
\end{tabular}

$\mathrm{Kd}=$ Coeficiente de sorção.

Koc = Coeficiente de sorção normalizado para carbono orgânico do solo.

Foi verificado que aumentando-se o pH do solo os coeficientes de sorção Kd e Koc diminuem. Sabe-se que a sorção do 2,4-D, composto ácido com constante de ionização (pKa) de 2,8, é principalmente determinada pela sua dissociação. Entretanto, sua dissociação pode não ser suficiente para explicar a variação em $\mathrm{Kd}$ (ou Koc) em função do $\mathrm{pH}$.

A sorção de formas aniônicas do pesticida pode, teoricamente, ocorrer em minerais com capacidade de troca aniônica. Embora a maioria dos solos apresente carga líquida negativa (devido à relativa abundância de cargas negativas sobre alumino-silicatos cristalinos e matéria orgânica), solos 
compostos principalmente de alumino-silicatos amorfos ou óxidos (hidróxidos, oxihidróxidos) de ferro e alumínio (como os Latossolos) podem apresentar carga líquida positiva em $\mathrm{pH}$ baixo.

A acessibilidade de grupos funcionais orgânicos do solo, capazes de interagir com o pesticida em função da dissociação da matéria orgânica (mudanças conformacionais), tem sido proposta para explicar diferenças observadas na sorção. Em pH baixo, a matéria orgânica do solo está na forma floculada e, portanto, o pesticida tem que se difundir por meio dela até os sítios de sorção (BRUSSEAU e RAO, 1989).

Se por um lado parece que a sorção das formas aniônicas de pesticidas ácidos tem sido superestimada, por outro, o efeito da dissociação da matéria orgânica por mudanças no pH do solo tem sido subestimada. JAFVERT (1990), LEE et al. (1990) e FONTAINE et al. (1991) propuseram explicações para a maior sorção que a esperada para compostos ácidos, particularmente em elevados níveis de pH. JAFVERT (1990) assumiu que a sorção da forma neutra não variava e que a sorção da forma aniônica apresentava dependência linear do pH. Considerou que a sorção da forma aniônica ocorreria principalmente em pH alto. Entretanto, com base no conhecimento atual não se espera sorção da forma aniônica quando há predominância de carga negativa nos solos. LEE et al. (1990) assumiram que a sorção da forma aniônica pode ocorrer pela formação de um par de íon neutro. Foi sugerido que a parte hidrofóbica do ânion orgânico poderia se adsorver à matéria orgânica do solo, com sua extremidade polar orientada para a fase aquosa mais polar. Entretanto, se esses processos realmente ocorrem podem não ser relevantes. Tem sido mostrado que a sorção de compostos ácidos decresce muito com o aumento do pH, quando a forma aniônica é dominante. FONTAINE et al. (1991) assumiram que a sorção das formas neutra e aniônica ocorrem apenas sobre a matéria orgânica. Entretanto, não se espera que haja sorção da forma aniônica do pesticida na matéria orgânica, exceto em níveis muito baixos de $\mathrm{pH}$, quando alguns grupos funcionais (como as aminas) tornam-se positivamente carregados. Assim, a contribuição da sorção da forma aniônica na sorção total de pesticidas ácidos, como o 2,4-D, ainda é questionável como regra geral. Estudos adicionais precisam ser realizados para explicar a grande variação entre os valores de Koc descritos na literatura e os encontrados neste trabalho.

\section{CONCLUSÃO}

Os resultados de Koc para o 2,4-D em solos brasileiros variaram até valores bem acima dos encontrados para o mesmo composto na literatura internacional. Observou-se tendência de aumento dos valores de Kd em solos com maiores teores de carbono orgânico, sendo verificada grande variação nos valores de Koc entre solos da mesma classe. Os valores de Kd e Koc diminuíram com o aumento do $\mathrm{pH}$. A dissociação do 2,4-D pode não ser suficiente para explicar a variação em Kd (ou Koc) em função do pH. Parece que a sorção das formas aniônicas de pesticidas ácidos tem sido superestimada em detrimento da consideração dos efeitos da dissociação da matéria orgânica, que também depende do $\mathrm{pH}$ do solo.

\section{ABSTRACT}

\section{SORPTION OF 2,4-D HERBICIDES IN BRAZILIAN SOILS}

This work aimed to generate and discuss data on acid pesticides sorption in Brazilian soils, taking into account the $\mathrm{pH}$ dependence. Soil samples were collected in different regions of Brazil and their properties were determined $(\%$ argila, $\% \mathrm{CO}$ and $\mathrm{pH}$. Sorption coefficient of 2,4-D were measured by means of a standard batch equilibrium method and high performance liquid chromatography (HPLC) analysis. The sorption coefficient (Kd) was estimated, and the organic carbon normalized sorption coefficient $(\mathrm{Koc})$ was calculated for each soil. The Koc results are between 21.3 and $306.5 \mathrm{~mL} / \mathrm{g}$, while the Koc values from international literature are in the 20 to $60 \mathrm{~mL} / \mathrm{g}$ range. The Koc values tend to increase in soils with large organic carbon content. However, great Koc values variation occurred among soils in the same class. Additionally, the effects of $\mathrm{pH}$ in 2,4-D sorption were studied in a Brazilian Oxisol. The Kd and Koc values decreased with $\mathrm{pH}$ increasing. Sorption of 2,4-D, as an acid compound, is mainly driven by its dissociation. However 
2,4- $\mathrm{D}$ dissociation is not enough to explain the variation in $\mathrm{Kd}$ (or $\mathrm{Koc}$ ) as a function of $\mathrm{pH}$. In general, anionic-form sorption of acidic pesticides has been overvalued; on the other hand, the overall effect of organic matter dissociation owing to $\mathrm{pH}$ changes has been overlooked.

KEY-WORDS: PESTICIDE-SORPTION; SOIL; ORGANIC MATTER; 2,4-D.

\section{REFERÊNCIAS}

ABERNATHY, J.R.; WAX, LM. Bentazon mobility and absorption in twelve Illinois soils. Weed Science, v.21, p.224227, 1973.

ALMENDROS, G. Sorptive interactions of pesticides in soils treated with modified humic acids. European Journal of Soil Science, v.46, p.287-301, 1995.

BRUSSEAU, M.L.; RAO, P.S.C. The influence of sorbate-organic matter interactions on sorption nonequilibrium. Chemosphere, v.18, p.1691-1706, 1989.

CAMARGO, O.A. de; MONIZ, A.C.; JORGE J.A.; VALADARES, J.M.A.S. Métodos de análise química, mineralógica e física de solos. Campinas: Instituto Agronômico, 1986. (IAC. Boletim Técnico, 106).

EMBRAPA. Centro Nacional de Pesquisa de Solos. Manual de métodos de análise de solo. 2. ed. Rio de Janeiro, 1997. 212 p.

FONTAINE, D.D.; LEHMAN, R.G.; MILLER, J.R. Soil adsorption of neutral and anionic forms of sulfonamide herbicide, flumetsulam. Journal of Environmental Quality, v.20, p.759-762, 1991.

GOETZ, A.J.; WALKER, R.H.; WEHTJE, G.; HAJEK, B.F. Sorption and mobility of chlorimuron in Alabama soils. Weed Science, v.37, p.428-433, 1989.

GRAHAM-BRYCE, I.J. The behaviour of pesticides in soil. In: GREENLAND, D.J.; HAYES, M.H.B. (Eds.). The chemistry of soil processes. New York: John Wiley \& Sons, 1981. p. 621-670.

GREY, T.L.; WEHTJE, G.R.; WALKER, R.H; HAJEK, B.H. Sorption and mobility of Bentazon in Coastal Plain soil. Weed Science, v.44, p.166-170, 1996.

HAIDER, K. Problems related to the humification processes in soils of temperate climates. In: STOTZBY, G.; BOLLAG, J.M. (Eds.). Soil biochemistry. New York: Marcel Dekker, 1992. v. 7, p. 55-94.

HAYES, M.H.B. Extraction of humic substances from soil. In: AIKEN, G.R.; MCKNIGHT, D.M.; WERSHAW, R.L.; MACCARTHY, P. (Ed.). Humic substances in soil, sediment, and water: geochemistry, isolation, and characterization. New York: John Wiley \& Sons, 1985. p. 329-362.

HORNSBY, A.G.; WAUCHOPE, R.D.; HERNER, A.E. Pesticide properties in the environment. New York: Springer-Verlag, 1996.

JAFVERT, C.T. Sorption of organic acid compounds to sediments: initial model development. Environmental Toxicology and Chemistry, v.9, p.1259-1268, 1990.

LEE, L.S.; RAO, P.S.C.; NKEDI-KIZZA, P.; DELFINO, J.J. Influence of solvent and sorbent characteristics on distribution of pentachlorophenol in octanol-water and soil-water systems. Environmental Science and Technology, v.24, p.654-661, 1990.

LI K.; LIU, W.; XU, D.; LEE, S. Influence of organic matter and pH on betazone sorption in soils. Journal of Agricultural and Food Chemistry, v.51, p.5362-5366, 2003.

NEARPASS, D.C. Adsorption of picloram by humic acids and humin. Soil Science, v.121, p.272-277, 1976.

OGRAM, A.V.; JESSUP, R.E.; OU, L.T.; RAO, P.S.C. Effects of sorption on biological degradation rates of (2,4dichlorophenoxy) acetic acid in soils. Applied Environmental Microbiology, v.49, p.582-587, 1985.

SCHEUNERT, I. Transformation and degradation of pesticides in soil. In: W. EBING (Ed.). Terrestrial behavior of pesticides. New York: Springer-Verlag, 1992. p.23-75. (Chem. Plant Prot. Ser. 8).

SCHNITZER, M. Soil organic matter: the next 75 years. Soil Science, v.151, p.41-58, 1991.

SCHNITZER, M.; KHAN, S.U. Soil organic matter. New York: Elsevier Sci., 1978.

SENESI, N. Binding mechanisms of pesticides to soil humic substances. Science of Total Environment, v.123/ 124, p.63-76, 1992 
SHEA, P.J. Chlorsulfuron dissociation and adsorption on selected adsorbents and soils. Weed Science, v.34, p.474-478, 1986. STEVENSON, F.J. Humus chemistry: genesis, composition, and reactions. New York: John Wiley \& Sons, 1982. TOMLIN, C.D.S. (Ed.). The pesticide manual. 11 ${ }^{\text {th }}$ ed. Farnham, Surrey, UK: British Crop Protection Council, 2000. WALKER, A.; COTTERHILL, E.G.; WELCH, S.J. Adsorption and degradation of Chlorsulfuron and Metsulfuron-metil in soils from different depths. Weed Research, v.29, p.281-287, 1989.

WERSHAW, R.L. A new model for humic materials and their interactions with hydrophobic organic chemicals in soil-water and sediment-water systems. Journal of Contaminant Hydrology, v.1, p.29-45, 1986. 